\title{
Liver Transplantation for Perihilar Cholangiocarcinoma in the Era of Transplant Oncology: Urgent Need for a Common Definition of Resectability, Transplantability, and Curability
}

\author{
Keita Shimata, Hidekazu Yamamoto, Yasuhiko Sugawara, Taizo Hibi
}

Department of Transplantation and Pediatric Surgery, Kumamoto University Hospital, Kumamoto, Japan

\section{ABSTRACT}

The indications for liver transplantation in hepatobiliary malignancies have been carefully expanded, giving rise to a new era of "Transplant Oncology," the fusion of transplantation medicine and oncology. Curative resection is the only curative therapy for perihilar cholangiocarcinoma. Liver transplantation may achieve a complete resection with adequate negative margins in patients with locally advanced, unresectable disease due to bilateral invasion of second-order biliary radicals, portal vein and/or hepatic artery involvement, or insufficient hepatic reserve. An aggressive multidisciplinary approach which consists of neoadjuvant chemoradiotherapy, followed by liver transplantation, was introduced by the Mayo Clinic group for selected patients with unresectable perihilar cholangiocarcinoma. This treatment strategy has become increasingly accepted in the U.S., with acceptable results. However, the definition of "unresectable disease" is not universal and therefore, the true survival benefit of liver transplantation, over extended liver resection combined with portal vein/hepatic arterial reconstruction, remains unclear. This review describes the history current controversies and future directions that need to be taken liver transplantation for perihilar cholangiocarcinoma.

Key words: perihilar cholangiocarcinoma, liver transplantation, neoadjuvant chemotherapy, transplant oncology

\section{HISTORY AND CURRENT STATUS OF LIVER TRANSPLANTATION FOR PERIHILAR CHOLANGIOCARCINOMA}

Complete removal of the tumor is critically important for cure of perihilar cholangiocarcinoma. Liver transplantation may allow a histologically curative resection with adequate negative margins in patients with locally advanced, unresectable disease due to bilateral invasion of second-order biliary radicals, portal vein and/or hepatic artery involvement, or insufficient hepatic reserve (1).

Since the late 1980s, liver transplantation for perihilar cholangiocarcinoma has been attempted with poor resultant. Pichlmayr et al. performed liver transplantation alone for patients with unresectable cholangiocarcinoma, proven by

\author{
Corresponding author: \\ Taizo Hibi, MD, PhD \\ Department of Transplantation \\ and Pediatric Surgery, Kumamoto \\ University Graduate School of \\ Medical Sciences \\ 1-1-1 Honjo, Chuo-ku, Kumamoto \\ 860-8556, Japan \\ Email: taizohibi@gmail.com
}

Received: 21.06.2018

Accepted: 15.07.2018 
surgical exploration, and reported 1-, 3-, and 5-year survival rates of $60 \%, 21.4 \%$, and $17.1 \%$, respectively (2). Iwatsuki et al. reported that the 1-, 3-, and 5 -year survival rates after liver transplantation were $59.3 \%, 36.2 \%$, and $36.2 \%$, respectively, and that liver transplantation for perihilar cholangiocarcinoma was unjustified given the 36\% 5-year survival after liver transplantation in patients with negative surgical margins and no nodal disease. The authors also mentioned that the role of liver transplantation for perihilar cholangiocarcinoma was limited because of the inefficient use of organs in the context of organ shortages (3). Therefore, most programs have abandoned liver transplantation alone for cholangiocarcinoma because of these discouraging results.

In 2000, the Mayo Clinic group developed a protocol using preoperative irradiation and chemotherapy for patients with perihilar cholangiocarcinoma. Patients initially received external-beam irradiation plus bolus fluorouracil (5-FU), followed by brachytherapy with iridium and concomitant protracted venous infusion of 5-FU. 5-FU was then administered continuously through an ambulatory infusion pump until liver transplantation. A total of 11 patients, out of the initial 19 patients who were enrolled onto the study, underwent liver transplantation. The study outcomes were encouraging (4). In 2005, the Mayo Clinic group reported on a modified protocol after introducing a novel neoadjuvant chemoradiotherapy regimen for unresectable perihilar cholangiocarcinoma (5). Patient eligibility and exclusion criteria for liver transplantation are described in table 1. Thirty-eight patients underwent liver transplantation and 1-, 3-, and 5-year survival rates were $92 \%, 82 \%$, and $82 \%$, respectively. The authors concluded that liver transplantation with neoadjuvant chemoradiation achieved better survival with lower recurrence rates than conventional resection and should be considered as an alternative to resection for patients with localized, node-negative perihilar cholangiocarcinoma (5). The multicenter retrospective study in 2012 across 12 U.S. institutions with variable neoadjuvant protocols also confirmed excellent outcomes of neoadjuvant chemoradiotherapy followed by liver transplantation for patients with unresectable perihilar cholangiocarcinoma (6).

At present, only one prospective, randomized, multicenter study, comparing liver transplantation preceded by neoadjuvant chemoradiotherapy to conventional liver and bile duct resection for resectable perihilar cholangiocarcinoma (Liver Resection Versus
Table 1 - Patient eligibility and exclusion criteria of the Mayo Clinic protocol

(adopted from: Rosen CB, Heimbach JK, Gores GJ. Liver transplantation for cholangiocarcinoma. Transpl Int 2010;23:692-7.)

Patient eligibility
Diagnosis of cholangiocarcinoma
Transluminal biopsy or brush cytology
Malignant appearing stricture and:
$\quad$ CA-19.9 $100 \mathrm{mg} / \mathrm{ml}$; or
Biliary ploidy by FISH; or
$\quad$ Mass at site of stricture
Unresectable tumor above cystic duct
Pancreatoduodenectomy for microscopic involvement of CBD
Resectable CCA arising in PSC
Absence of intra- and extrahepatic metastases
Candidate for liver transplantation
Exclusion criteria
Intrahepatic cholangiocarcinoma
Uncontrolled infection
Prior radiation or chemotherapy
Prior biliary resection or attempted resection
Intrahepatic metastases
Evidence of extrahepatic disease
History of other malignancy within 5 years
Transperitoneal biopsy or FNA (including EUS)
Radial diameter $>3 \mathrm{~cm}$

Radio-chemotherapy-Transplantation for Hilar Cholangiocarcinoma [TRANSPHIL] study), is underway in France (www.clinicaltrials.gov NCT02232932). Here, an interventional group receives liver transplantation preceded by neoadjuvant chemoradiotherapy (capecitabine; $800 \mathrm{mg} / \mathrm{m}^{2}$ twice a day for 5 weeks and external radiotherapy; a total of 50 gray delivered over 5 weeks). The control group receives conventional liver and bile duct resection. The primary endpoint for this study is overall survival at 5 years. The secondary endpoint is recurrence-free survival at 3 years, as evaluated by CT scan and tumor markers. This study should provide additional insights as to whether liver transplantation for perihilar cholangiocarcinoma is a more effective treatment than liver resection in patients who are candidates for resection.

\section{CLINICAL GUIDELINES}

Liver transplantation for perihilar cholangiocarcinoma is emerging concept, and there is wide discrepancy in its applicability among currently available clinical guidelines. Patients need to be carefully selected to avoid futile transplants in the face of critical organ shortage. Several proposed criteria are described below. 


\section{National Comprehensive Cancer Network Guidelines}

National Comprehensive Cancer Network guidelines version 1.2018 states that liver transplantation is a potentially curative option for selected patients with lymph node-negative, non-disseminated, locally advanced perihilar cholangiocarcinoma, and with either unresectable disease with normal biliary and hepatic function, or with underlying chronic liver disease precluding surgery. Based on the United Network for Organ Sharing database, Becker et al. reported that the 5 -year survival rate was $45 \%$ for patients with diagnosed cholangiocarcinoma transplanted between 1995 and 2005. They advocated that the outcomes following liver transplantation for cholangiocarcinoma had improved during the recent era of transplantation and that the factors contributing to improved outcomes included more precise staging, more restricted patient selection, and more effective adjuvant therapies (7). As previously mentioned, Darwish et al. reported the first multicenter study to examine the effectiveness of combining preoperative chemoradiataion and liver transplantation for highly selected patients with locally advanced, unresectable perihilar cholangiocarcinoma. Recurrencefree survival rates were $78 \%, 65 \%$ and $59 \%$ at 2,5 and 10 years respectively. The outcomes were not only similar to results from previous single-center series $(5,8)$ but also similar to liver transplantation outcomes for other malignant and non-malignant indications (6). There are many reports advocating the efficacy of liver transplantation for selected patients with perihilar cholangiocarcinoma but these data are insufficient to achieve commonly accepted criteria in the guidelines. The panel encourages continuation of clinical research in this area, and recommends referral to a transplant center if patient is a potential transplant candidate.

\section{ESMO Clinical Practice Guidelines}

The ESMO clinical practice guidelines describe liver transplantation in locally unresectable cases involving a multidisciplinary approach including neoadjuvant chemoradiotherapy followed by liver transplantation. However, this treatment has not become the standard of care. In 2016, Mantel et al. reported the importance of appropriate patient selection on liver transplantation for perihilar cholangiocarcinoma outcomes using the European Liver Transplant Registry. In this retrospective study, patients who complied with the Mayo Clinic criteria (group A) showed a significantly better survival compared to patients who did not meet the criteria (group B). The 5 -year survival rate was $59 \%$ for group A versus $21 \%$ for group $B(P=0.001)(9)$. The ongoing French phase III TRANSPHIL trial should help determine whether this treatment should be the standard treatment for cholangiocarcinoma.

\section{Japanese Clinical Practice Guidelines}

There is no statement about liver transplantation for cholangiocarcinoma in the Japanese clinical practice guidelines. Some Japanese hepatobiliary surgeons have emphasized that most patients with early-stage, unresectable cancer (i.e., good candidates for transplantation according to the Mayo Clinic criteria) have resectable tumors, and that extended resections for such patients can produce favorable outcomes (10-13). The guidelines state that biliary tract cancers with metastases to the liver, lungs, bones, peritoneum, and distant lymph nodes (para-aorta or extra-abdominal lymph nodes) are contraindications for surgical resection; however, there is no consensus on criteria to define locally unresectable disease (14).

\section{RESECTABILILTY, TRANSPLANTABILITY, AND CURABILITY}

As shown above, neoadjuvant therapy, followed by liver transplantation, is reserved for a highly selected group of patients (including those with tumors $<3 \mathrm{~cm}$ in size and no lymph node involvement) with unresectable disease and patients with underlying primary sclerosing cholangitis (15). However, the definition of "unresectable disease" remains highly problematic. Bismuth defined type IV lesions as unresectable (16). The Mayo Clinic criteria for unresectability include bilateral segmental ductal extension, encasement of the main portal vein and unilateral segmental ductal extension with contralateral vascular encasement. On the other hand, Nagino et al. reported on 73 patients with Bismuth type IV tumors and/or who underwent combined vascular resection, and thereby met the unresectability criteria. Their 5 -year survival rate was $60.4 \%$, similar to the survival for the transplant patients with de novo cancer. Nagino et al. stated that transplants are unnecessary in most patients in the Mayo series and that surgical treatment for perihilar cholangiocarcinoma was evolving steadily, with expanded surgical indications, decreased mortality, and increased survival (10). More recently, Ebata et al. reported outcomes of patients with Bismuth type IV tumors who underwent surgical resection. Of the 332 patients with type IV tumors, 216 (65.1\%) underwent surgical resection. The 
5 -year survival rate of $53 \%$ of patients with pNOMO disease in their series was similar to that of patients with de novo cancer who received liver transplantation. They also insisted that most patients with early-stage cancer, considered unresectable based on Mayo Clinic criteria who were good candidates for transplantation, had resectable tumors (17). This highlights the need of a consensus for the definition of "unresectable disease" before the role of liver transplantation for curative treatment of this disease can be determined.

Recently, in addition to the prospective trial in France, the propriety of liver transplantation for patients with resectable cholangiocarcinoma has been discussed in the U.S. Ethun et al. evaluated the type of surgery (resection versus liver transplantation) for patients with perihilar cholangiocarcinoma using the database of the U.S. Extrahepatic Biliary Malignancy Consortium. They found that resection for patients with perihilar cholangiocarcinoma that met criteria for liver transplantation ( $<3 \mathrm{~cm}$ and lymph node-negative disease) resulted in significantly worse prognoses, compared to those who received neoadjuvant therapy followed by liver transplantation for the same criteria with unresectable disease, when performing intention-to-treat analyses ( $54 \%$ versus $29 \%$ at 5 years, $P=0.03$ ) (18). (18). Rosen wrote in his editorial that neoadjuvant therapy and liver transplantation "should not be done in lieu of resection for potentially resectable cholangiocarcinoma," because the benefit is too low to justify use of a deceased or living donor liver. In the study, the survival benefit of neoadjuvant therapy and transplantation was only $14 \%$ when only patients with non-primary sclerosing cholangitis were compared, well below the minimal benefit commonly applied to transplantation; $50 \%$ survival at 5 years (19). Moreover, Nagino raised two issues in his editorial: i) The surgical outcomes of the resection group in the study were poor compared to the reports from Japan and Korea, and ii) Most tumors that met the Mayo Clinic criteria for liver transplantation, i.e., early-stage but unresectable cancer, may become resectable by exhausting all surgical options for perihilar cholangiocarcinoma, such as extended hepatic resections including trisectionectomies combined with major vascular resections, and hepatopancreatoduodenectomy (20). Whether there would be a significant survival benefit with liver transplantation over resection for resectable disease remains unclear and it is of paramount importance to discuss resectability, transplantability, and, most importantly, curability in an international framework.

\section{FUTURE PERSPECTIVES}

The indications of liver transplantation for perihilar cholangiocarcinoma have been carefully expanded by applying an aggressive multidisciplinary approach. This evolution shed light on the ambiguity of "unresectable disease," and therefore, the true survival benefit of liver transplantation remains unclear. Nonetheless, liver transplantation may potentially change the landscape of treatment for perihilar cholangiocarcinoma and we should hold broad, international discussions to establish a consensus on resectability, transplantability, and curability by integrating the growing body of evidence in the context of transplant oncology (21).

In conclusion, although neoadjuvant therapy followed by liver transplantation for perihilar cholangiocarcinoma has proven to be a highly effective strategy for cholangiocarcinoma, there is an urgent need to build a common language for this surgically curable disease. Once a consensus is reached among an international group of experts, prospective studies of how to optimize oncological treatments will be worthwhile.

\section{Conflicts of interest}

The authors declare that there are no conflicts of interest.

\section{REFERRENECES}

1. Goldaracena N, Gorgen A, Sapisochin G. Current status of liver transplantation for cholangiocarcinoma. Liver Transpl. 2018; 24(2):294-303.

2. Pichlmayr R, Weimann A, Tusch G, Schlitt HJ. Indications and Role of Liver Transplantation for Malignant Tumors. Oncologist. 1997; 2(3):164-70.

3. Iwatsuki S, Todo S, Marsh JW, Madariaga JR, Lee RG, Dvorchik I, et al. Treatment of hilar cholangiocarcinoma (Klatskin tumors) with hepatic resection or transplantation. J Am Coll Surg. 1998; 187(4):358-64.

4. De Vreede I, Steers JL, Burch PA, Rosen CB, Gunderson LL, Haddock MG, et al. Prolonged disease-free survival after orthotopic liver transplantation plus adjuvant chemoirradiation for cholangiocarcinoma. Liver Transpl. 2000;6(3):309-16.

5. Rea DJ, Heimbach JK, Rosen CB, Haddock MG, Alberts SR, Kremers WK, et al. Liver transplantation with neoadjuvant chemoradiation is more effective than resection for hilar cholangiocarcinoma. Ann Surg. 2005;242(3):451-8; discussion 8-61.

6. Darwish Murad S, Kim WR, Harnois DM, Douglas DD, Burton J, Kulik LM, et al. Efficacy of neoadjuvant chemoradiation, followed by liver transplantation, for perihilar cholangiocarcinoma at 12 US centers. Gastroenterology. 2012;143(1):88-98.e3; quiz e14.

7. Becker NS, Rodriguez JA, Barshes NR, O'Mahony CA, Goss $\mathrm{JA}$, Aloia TA. Outcomes analysis for 280 patients with cholangiocarcinoma treated with liver transplantation over an 18-year period. J Gastrointest Surg. 2008;12(1):117-22. 
8. Heimbach JK, Gores GJ, Haddock MG, Alberts SR, Pedersen R, Kremers W, et al. Predictors of disease recurrence following neoadjuvant chemoradiotherapy and liver transplantation for unresectable perihilar cholangiocarcinoma. Transplantation. 2006;82(12):1703-7.

9. Mantel HT, Westerkamp AC, Adam R, Bennet WF, Seehofer D, Settmacher U, et al. Strict Selection Alone of Patients Undergoing Liver Transplantation for Hilar Cholangiocarcinoma Is Associated with Improved Survival. PLoS One. 2016;11(6):e0156127.

10. Nagino M, Ebata T, Yokoyama Y, Igami T, Sugawara G, Takahashi Y, et al. Evolution of surgical treatment for perihilar cholangiocarcinoma: a single-center 34 -year review of 574 consecutive resections. Ann Surg. 2013;258(1):129-40.

11. Ebata T, Yokoyama Y, Igami T, Sugawara G, Takahashi Y, Nimura Y, et al. Hepatopancreatoduodenectomy for cholangiocarcinoma: a single-center review of 85 consecutive patients. Ann Surg. 2012; 256(2):297-305.

12. Nagino M, Nimura $Y$, Nishio H, Ebata T, Igami T, Matsushita M, et al. Hepatectomy with simultaneous resection of the portal vein and hepatic artery for advanced perihilar cholangiocarcinoma: an audit of 50 consecutive cases. Ann Surg. 2010;252(1):115-23.

13. Shimizu H, Kimura F, Yoshidome H, Ohtsuka M, Kato A, Yoshitomi $\mathrm{H}$, et al. Aggressive surgical resection for hilar cholangiocarcinoma of the left-side predominance: radicality and safety of left-sided hepatectomy. Ann Surg. 2010;251(2):281-6.

14. Miyazaki M, Yoshitomi H, Miyakawa S, Uesaka K, Unno M, Endo I, et al. Clinical practice guidelines for the management of biliary tract cancers 2015: the 2nd English edition. J Hepatobiliary Pancreat Sci. 2015;22(4):249-73.

15. Mansour JC, Aloia TA, Crane CH, Heimbach JK, Nagino M, Vauthey JN. Hilar cholangiocarcinoma: expert consensus statement. HPB (Oxford). 2015;17(8):691-9.

16. Bismuth $\mathrm{H}$, Nakache R, Diamond T. Management strategies in resection for hilar cholangiocarcinoma. Ann Surg. 1992;215(1):31-8.

17. Ebata T. Surgical resection for Bismush type IV perihilar cholangiocarcinoma. BJS. 2018;105:829-38.

18. Ethum C. Transplantation Versus Resection for Hilar Cholangiocarcinoma An Argument for Shifting Treatment Pradigms for Resectable Disease. Ann Surg. 2018;267:797-805.

19. Rosen CB. Transplantation Versus Resection for Hilar Cholangiocarcinoma: An Argument for Shifting Paradigms for Resectable Disease in Annals of Surgery 2018. Ann Surg. 2018; 267(5): 808-9.

20. Nagino M. Surgical Treatment of Perihilar Cholangiocarcinoma. Ann Surg. 2018;267:806-7.

21. Hibi T, Itano 0 , Shinoda M, Kitagawa Y. Liver transplantation for hepatobiliary malignancies: a new era of "Transplant Oncology" has begun. Surg Today. 2017;47(4):403-15. 